\title{
Norois
}

Environnement, aménagement, société

\section{Le rechargement de la plage de Sainte-Anne-du- Portzic (Brest-Finistère) : quand la recherche fondamentale se met au service d'une gestion cohérente du littoral}

Replenishment of the beach of Sainte-Anne-du-Portzic (Brest-Finistère): when fundamental research serves a comprehensive coastal management

\section{Bernard Fichaut et Serge Suanez}

\section{OpenEdition Journals}

\section{Édition électronique}

URL : http://journals.openedition.org/norois/2175

DOI : $10.4000 /$ norois. 2175

ISBN : 978-2-7535-1556-7

ISSN : 1760-8546

Éditeur

Presses universitaires de Rennes

\section{Édition imprimée}

Date de publication : 1 novembre 2008

Pagination : 29-44

ISBN : 978-2-7535-0788-3

ISSN : 0029-182X

Référence électronique

Bernard Fichaut et Serge Suanez, «Le rechargement de la plage de Sainte-Anne-du-Portzic (BrestFinistère) : quand la recherche fondamentale se met au service d'une gestion cohérente du littoral », Norois [En ligne], 208 | 2008/3, mis en ligne le 01 novembre 2010, consulté le 19 avril 2019. URL : http://journals.openedition.org/norois/2175; DOI : 10.4000/norois.2175 


\title{
Le rechargement de la Plage de Sainte-Anne-du-Portzic (BREST-FINISTÈRE) : QUAND LA RECHERCHE FONDAMENTALE SE MET AU SERVICE D'UNE GESTION COHÉRENTE DU LITTORAL
}

\author{
Bernard Fichaut, Serge Suanez \\ GEOMER - UMR 6554 LETG CNRS \\ (Université de Bretagne Occidentale), \\ IUEM, Technopôle Brest-Iroise, place Nicolas-Copernic - 29280 Plouzané, France \\ bernard.fichaut@univ-brest.fr, serge.suanez@univ-brest.fr
}

\begin{abstract}
RÉSUMÉ
La plage de Sainte-Anne-du-Portzic était la seule plage naturellement sableuse de l'agglomération brestoise. La disparition graduelle du sable au cours de la seconde moitié du $\mathrm{XX}$ siècle a entraîné une baisse de l'attractivité de cette plage initialement très fréquentée. Cet article expose la méthodologie de l'étude de faisabilité d'un rechargement. L'analyse diachronique de photographies obliques et l'étude de l'historique des aménagements successifs a permis de déterminer l'origine du désensablement du haut de plage. Il résulte à la fois de la durcification progressive du trait de côte qui a augmenté la réflexivité du haut de l'estran, et de la construction d'une jetée qui a modifié l'hydrodynamisme du secteur. Les mesures de terrain au DGPS, sur une année entière, montrent qu'à l'heure actuelle les transferts sédimentaires sont extrêmement limités et ne se font jamais vers le haut de plage. L'analyse granulométrique montre que le sable qui a disparu du haut d'estran est stocké en bas de la zone intertidale et pourra être utilisé pour le rechargement. Le volume requis a été déterminé par modélisation de la topographie future de la plage. L'opération de rechargement ne pourra être menée qu'après reprise d'une partie des aménagements qui au fil du temps ont modifié la dynamique hydrosédimentaire de la plage.
\end{abstract}

MOTS CLÉ : DGPS - rade de Brest - rechargement - photo-interprétation - transport sédimentaire

\footnotetext{
ABSTRACT

Replenishment of the beach of Sainte-Anne-du-Portzic (Brest-Finistère): when fundamental research serves a comprehensive coastal management

The beach of Sainte-Anne-du-Portzic used to be the sole sandy beach in the vicinity of Brest. During the second half of $20^{\text {th }}$ century the gradual disappearance of sand led to a decline in attendance of this once highly praised beach. This paper presents the methodology of the feasibility study of beach replenishment. The diachronic analysis of oblique photographies and the tracing of the history of the set up of structures enabled to determine the origin of sand loss on the upper part of the beach. It is the result of the edification of man made structures that increased the reflexivity of the upper beach and of the construction of a jetty that has modified local hydrodynamism. DGPS field measurements, during an entire year, show that presently
} 
sedimentary movements are limited and never occur toward the upper beach. Granulometric analysis demonstrates that the sand has been relocated at the base of the beach and is likely to be used for replenishment. This operation will occur only once some of the structures responsible for the changes in the hydrodynamics of the beach will have been modified.

KEY WORDS : bay of Brest - beach replenishment - DGPS - photograph analysis - sediment transport

La plage de Sainte-Anne-du-Portzic, située au contact des communes de Brest et de Plouzané, est la seule plage naturellement sableuse de la rade de Brest (fig. 1). Située à seulement 8 kilomètres du centre-ville, et desservie dès le début du XX ${ }^{e}$ siècle par les transports en commun, elle est devenue la plage favorite des brestois et a vu sa fréquentation augmenter lorsque les autres plages de Brest ont été remblayées pour accueillir les extensions du port dans la première moitié du siècle. Après la seconde guerre mondiale, en dépit de l'avènement de la voiture individuelle et de l'accès facilité aux autres plages de la région elle est restée très prisée en particulier auprès des habitants des quartiers populaires de la partie occidentale de la ville. Aujourd'hui l'extension de Brest la positionne à proximité immédiate de quartiers résidentiels en pleine expansion.

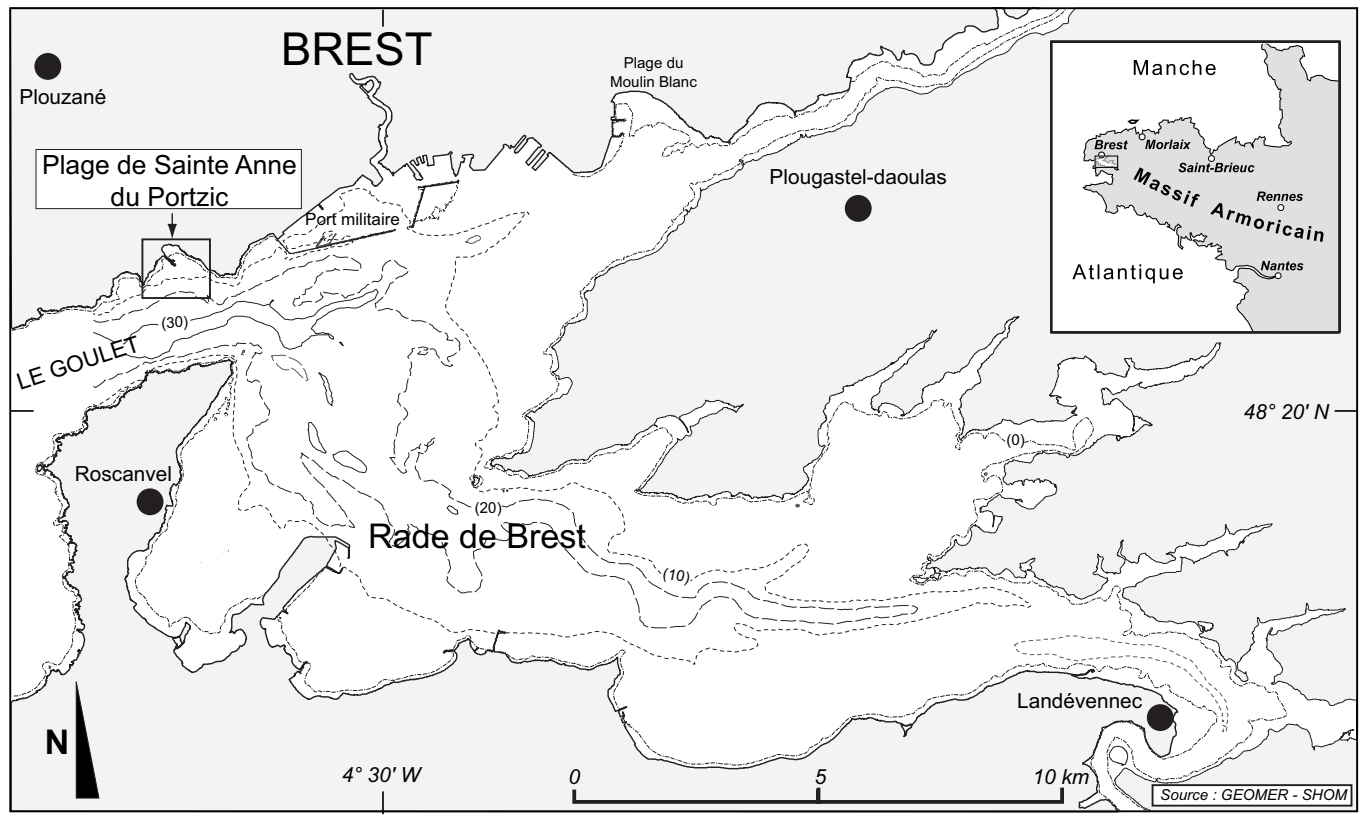

Figure 1 : Carte de localisation

Location map

Toutefois, depuis plus de vingt ans, à la suite d'une série d'aménagements affectant le trait de côte et les abords de l'anse, la plage n'a cessé de perdre du sable et elle est aujourd'hui essentiellement caillouteuse. À marée haute seul le sud ouest est hors d'eau et par fort coefficient seules quelques dizaines de mètres carrés d'estran restent émergés. L'étude présentée ici a été réalisée, à la demande de la Direction de la Protection du Patrimoine Naturel et Sensible (DPPNS), service en charge des plages au sein de Brest Métropole Océane (BMO). L'objectif premier de l'étude était d'analyser les modalités d'un rechargement de la plage. 
Le rechargement est une technique de protection qui connaît un engouement croissant (Rogers, 2000; Hamm et al., 2002; Matias et al., 2005; Finkl et Walker, 2005), car elle offre le double avantage d'être à la fois considérée comme une technique douce et d'augmenter potentiellement l'attractivité balnéaire des plages (Finkj, 1996; French, 2001 ; Cohen et Anthony, 2005). Classiquement, on estime qu'un rechargement ne peut fonctionner que si le sédiment rapporté est de même granulométrie, voire légèrement plus grossier que l'existant (Berg et Duane, 1968; Newman, 1976), même si cela diminue dans certains cas la valeur récréative des plages (Capobianco et al., 2002). Il s'avère que cette condition, même si elle paraît logique, n'assure en rien le succès d'une opération de ce type (Pilkey et Clayton, 1987 ; Pilkey, 1990; Hanson et al., 2002). Conventionnellement aussi, on estime que le volume de rechargement doit être supérieur au volume nécessaire qui a été calculé. En général au Pays-Bas les quantités rapportées dépassent de $20 \%$ les volumes requis, certains comme H. J. Verhagen (1996) estiment même qu'un dépassement de $40 \%$ doit être envisagé pour pallier les effets de réajustement du profil et les pertes latérales. Ces préconisations semblent empreintes de bon sens, mais ne garantissent en rien le succès de ces opérations. Les études de synthèse de 0 . Pilkey et T. D. Clayton (1987), K. Dixon et O. Pilkey (1989), et de L. Leonard et al. (1990a, 1990b), portant sur plus de 200 plages de la côte est et du Golfe du Mexique aux États-Unis d'Amérique, montrent que dans la plupart des cas, l'essentiel du matériel rapporté, parfois plusieurs millions de $\mathrm{m}^{3}$, est érodé dans les quelques mois qui suivent le rechargement artificiel. Les recherches menées à l'échelle du littoral européen (Hamm et al., 2002; Hanson et al., 2002), et plus spécifiquement aux Pays-Bas (van der Wal, 2004), donnent des conclusions similaires (van der Wal, 2004). Une grande incertitude pèse encore sur les raisons précises de ces échecs, mais les auteurs soulignent que la prise en compte des seuls paramètres granulométriques et volumétriques en est bien souvent la cause. À leurs yeux, la compréhension de la dynamique hydrosédimentaire du secteur littoral concerné couplée à une reconstitution de l'historique des causes du déséquilibre sédimentaire, constitue un préalable indispensable à une opération de rechargement (Capobianco et al., 2002; Finkl et Walker, 2005). Elle ne constitue pas pour autant une garantie de réussite.

À Sainte-Anne, au cours des années passées, la municipalité de Brest a effectué à plusieurs reprises des opérations de rechargement portant sur quelques dizaines de $\mathrm{m}^{3}$ de sable à chaque fois. Le sable était prélevé en bas d'estran et étalé en haut de plage sans qu'aucun résultat durable ne soit perceptible. Lors de la commande de l'étude, l'idée initiale de BMO était d'opérer un chargement beaucoup plus volumineux avec du sable obtenu par dragage des bancs sous-marins de Bertheaume et du Minou, situés par des fonds de moins de 20 mètres au débouché occidental du goulet de Brest. Cette source avait déjà été utilisée en 1978 pour la création de la plage artificielle du Moulin-Blanc à l'est de l'agglomération (Hallégouet et Guilcher, 1990). À cette occasion, $166000 \mathrm{~m}^{3}$ de sable avaient été prélevés (Pupier-Dauchez, 2002), sans qu'aucune étude ne soit menée pour estimer les effets de cette opération sur les plages environnantes. Aujourd'hui encore, ces gisements de sable sont exploités sans la moindre étude d'impact. D'emblée, notre travail s'est inscrit en rupture avec ce type d'approche et dans une optique résolument environnementaliste. Basée sur la compréhension de la dynamique hydrosédimentaire passée et actuelle de la plage, notre étude s'est faite en plusieurs étapes. Dans un premier temps, l'historique de l'évolution des aménagements et des modifications sédimentologiques induites a été établi à partir de l'analyse diachronique de photographies obliques prises au cours du siècle écoulé. Dans un deuxième temps, l'analyse de la morphodynamique actuelle a reposé sur une série de levés topographiques au DGPS entre avril 2007 et mars 2008. Enfin la proposition de solutions visant à permettre un réensablement durable de la plage a nécessité une caractérisation fine de la sédimentologie et de la granulométrie de l'estran (Fichaut et al., 2008).

\section{La durcification du trait de côte au début du vingtième siècle}

Jusqu'au début du vingtième siècle, la plage de Sainte-Anne-du-Portzic était vierge d'aménagement. Au sud ouest, le haut d'estran était constitué d'un cordon de galets barrant l'aval d'une petite vallée et protégeant des prairies de fauche situées en arrière. Le ruisseau de la vallée, marquant la 
limite entre les communes de Brest et de Plouzané, débouchait sur la plage à travers le cordon dont le revers servait de voie charretière. Au nord, de petites grèves de galets ourlaient la base de falaises (photo 1A). Sous le niveau des hautes mers de mortes eaux tout le reste de l'estran était sableux, à l'exception de quelques saillants rocheux de la plateforme d'abrasion apparaissant çà et là. Avant la première guerre mondiale, le développement du tourisme balnéaire et la construction d'une ligne de tramway dont le terminus se situait à moins d'un kilomètre de la plage vont entraîner les premiers aménagements importants. En quelques années, plusieurs hôtels, restaurants, dancings sont construits en front de mer au nord dans le secteur des falaises. Des terrasses accueillant buvettes, cabines de bain, etc., sont édifiées en avant de la falaise et protégées par des perrés maçonnés hauts de plusieurs mètres. La partie méridionale de la plage conserve son aspect antérieur (photo 1A). Dans l'entre-deux-guerres l'extension du port militaire vers l'ouest, sur la rive nord de la rade de Brest, entraîne la disparition par remblaiement de bon nombre de plages proches de la ville, et la plage de Sainte-Anne connaît une fréquentation accrue. Immédiatement après la seconde guerre mondiale, une route est édifiée au sommet du cordon de galets. Du côté brestois, tous les galets sont prélevés pour lui servir d'assise et un perré vertical en béton est édifié (photo 1B). Le reste du cordon conserve sa morphologie originelle jusqu'en 1963 où le mur est prolongé vers Plouzané. Là encore un volume important de galets est utilisé pour le remblais. L'abaissement du haut de plage renforcée par la réflexion des houles va entraîner le déchaussement de l'ouvrage dont la base sera renforcée par une banquette de béton. Ces modifications n'ont aucune conséquence sur la fréquentation de la plage rendue plus attractive encore du fait de l'augmentation, dans un premier temps, des superficies sableuses. Vers 1970, le débouché du ruisseau est busé et déporté vers le niveau de la mi-marée.

\section{La jetée IFREMER fait disparaître le sable}

En 1968, IFREMER s'implante sur le site de Beg an Nevent (la pointe du Diable) immédiatement au sud du site. Une jetée longue de 350 mètres est édifiée perpendiculairement à l'axe du Goulet. Initialement conçue pour accueillir des navires océanographiques, elle ne sera jamais utilisée à cet effet, mais elle va entraîner un bouleversement radical de l'hydrodynamisme, de la sédimentologie de l'estran et de l'attractivité de la plage qui va voir sa fréquentation baisser considérablement.

Comme l'ont montré plusieurs auteurs (Guilcher et al., 1957 ; SAUM, 1977), initialement, les houles provenant de l'ouest et réfractées sur la rive nord du Goulet, déferlaient parallèlement au trait de côte de la plage de Sainte-Anne (fig. 2A). L'orientation des rides de sable visibles sur tous les clichés antérieurs à la construction de l'ouvrage en atteste (photo 1A et B). Bien qu'aucune mesure ancienne n'existe, il est vraisemblable que les profils de la plage variaient au gré des états de la mer, une partie du stock de sable migrant alternativement vers les parties hautes et basses de l'estran. La présence de la jetée a deux effets conjugués. D’une part la diffraction des houles à son extrémité s'accompagne d'une rotation des crêtes qui déferlent obliquement à la côte dans le fond de l'anse et génèrent une dérive littorale du nord est vers le sud ouest (fig. 2 B). D'autre part l'amortissement des houles diminue considérablement leur compétence en particulier immédiatement en abri de la partie proximale de la jetée qui constitue l'aboutissement de la dérive. Depuis la construction de l'ouvrage la sédimentologie de l'anse a été profondément modifiée. Alors qu'antérieurement, la plage était entièrement recouverte de sable, actuellement il se cantonne en haut d'estran au sud ouest, et sous le niveau des basses mers moyennes essentiellement en zone subtidale. Ailleurs l'estran est rocheux ou couvert d'un pavage de cailloux et blocs anguleux exhumé après le départ du sable entraîné par la dérive littorale (photo $1 \mathrm{C}$ et fig. 3).

La seule présence de la jetée ne peut expliquer des changements sédimentologiques d'une telle ampleur. Si elle avait été le seul aménagement réalisé, il est fort probable qu'à l’heure actuelle, la plage serait simplement décalée vers le sud ouest, face aux houles diffractées. La migration du 

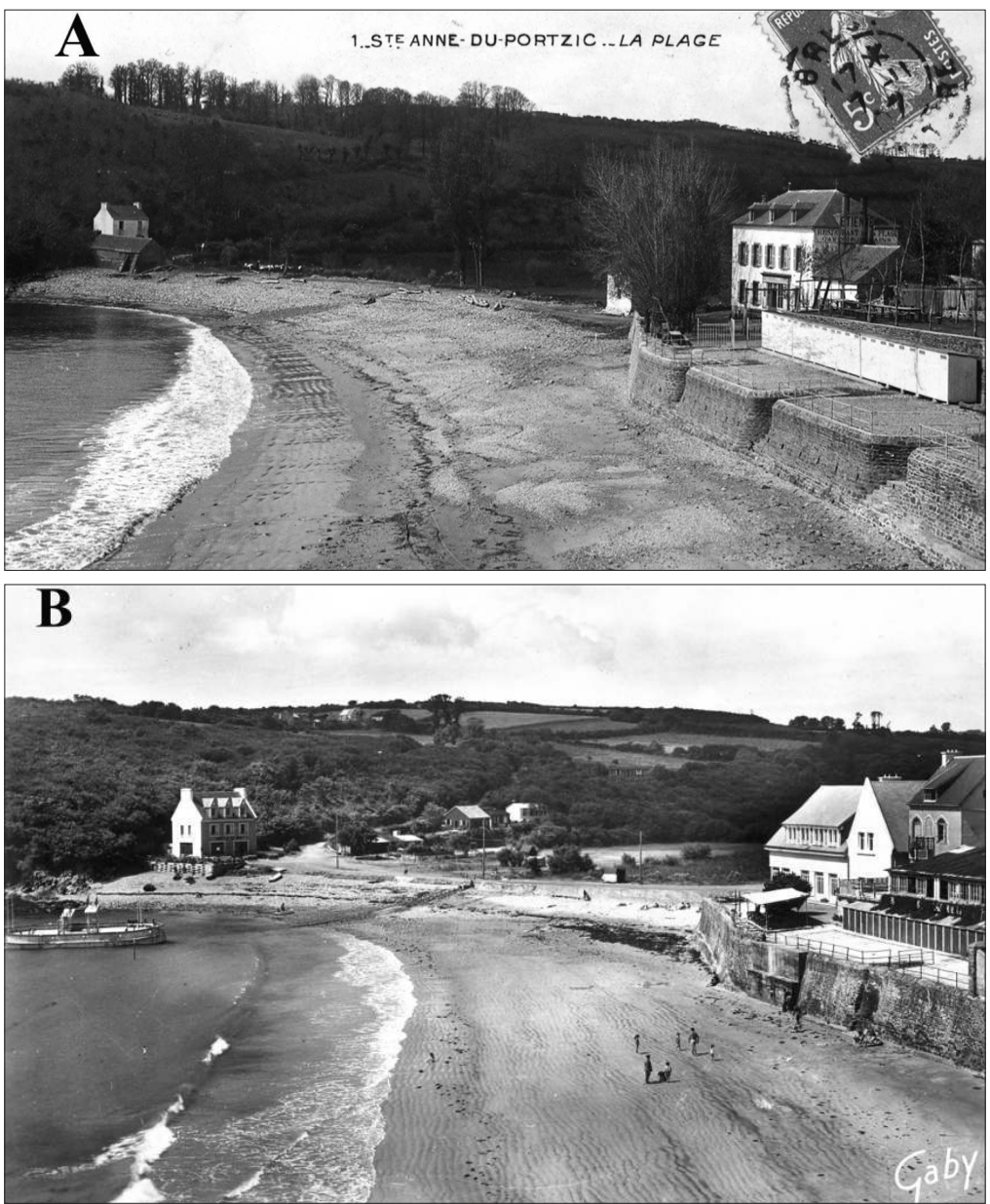

Photo 1 : La plage de Sainte-Anne-du-Portzic vue vers l'ouest : (A) vers $1910,(\mathrm{~B})$ à la fin des années 1950, (C) en décembre 2007

Westward view of the beach of SainteAnne-du-Portzic : (A) circa 1910 (B) by the end of years 1950 (C) in december 2007

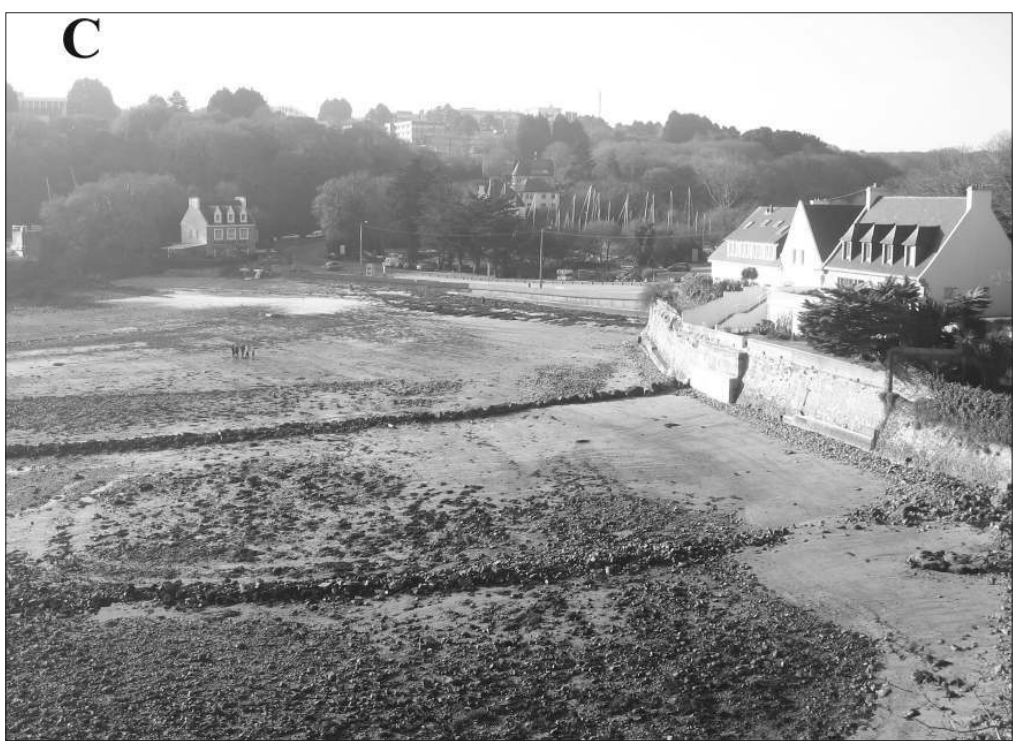




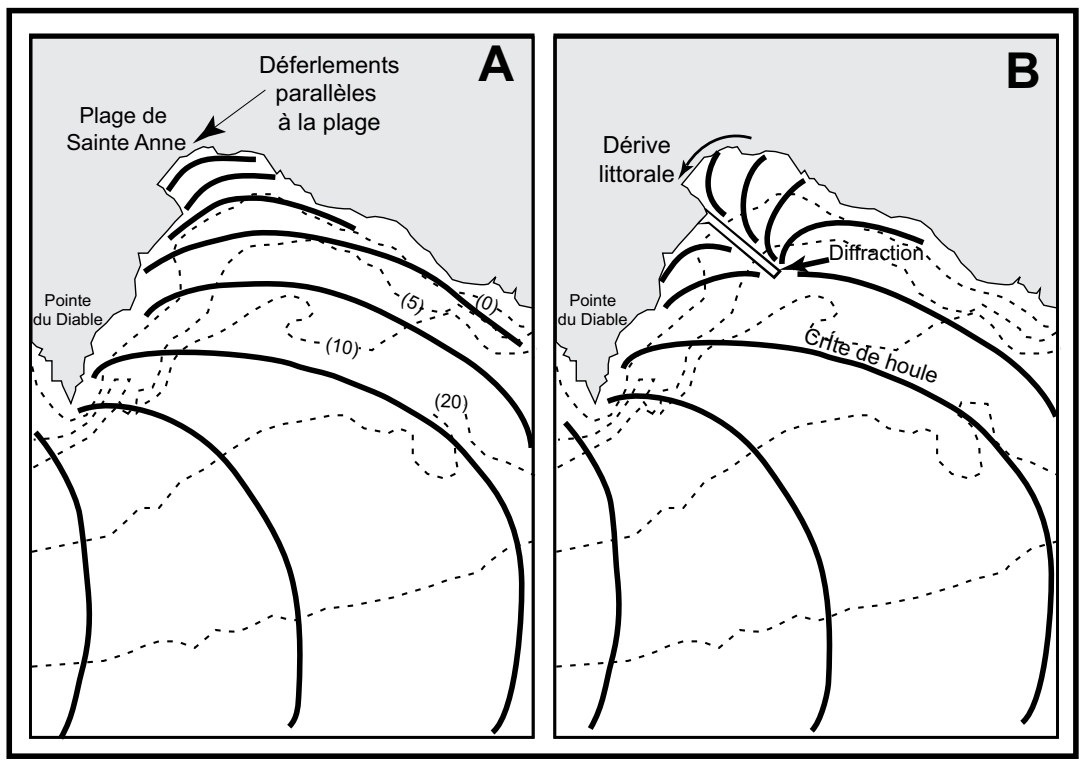

Figure 2 : Plan de vagues dans l'anse de Sainte-Anne réalisé par photointerprétation : (A) avant la construction de la jetée d'IFREMER, (B) après sa construction Wave refraction diagram in the bay of Sainte-Anne achieved using aerial photographs: (A) before the construction of IFRE$M E R$ jetty, (B) after construction

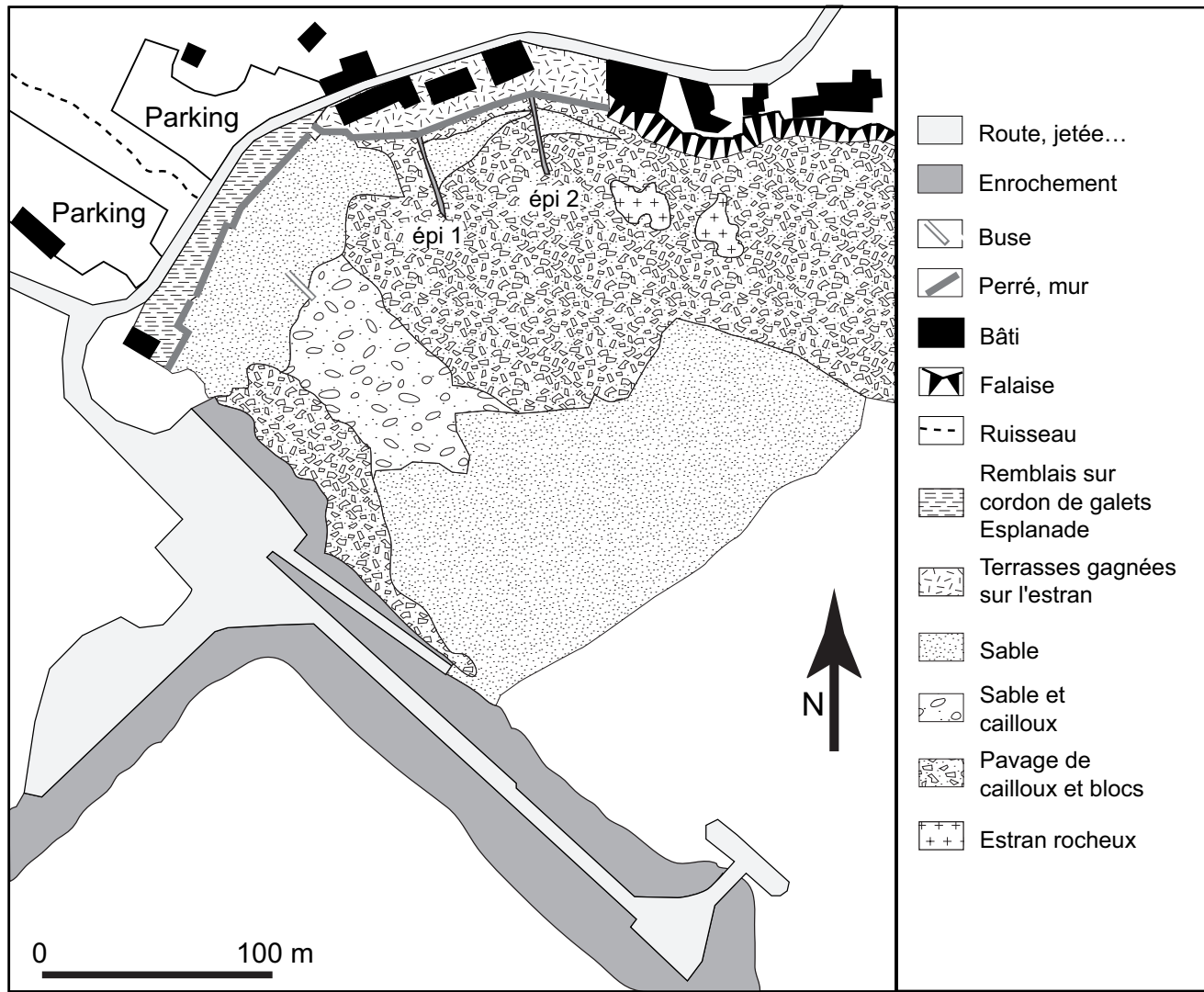

Figure 3 : Sédimentologie et aménagement de la plage de Sainte-Anne Sedimentology and equipment of the Sainte-Anne Beach 
stock sableux vers le bas de l'estran s'explique aussi par les effets des aménagements réalisés avant la construction de la jetée, effets qui n'ont eu d'impact irréversible qu'après l'édification de cette dernière. Le ruisseau débouchant sur la plage a toujours entraîné un départ de sable vers le bas de plage. La réflexion des vagues sur le mur du haut de plage ajoutée au busage de l'embouchure du ruisseau, a eu pour effet de renforcer ce phénomène et de déplacer le point de départ du sable plus bas sur l'estran. Toutefois, en l'absence de jetée, certaines houles étaient susceptibles de remonter occasionnellement le sable vers le haut de la plage. Actuellement, ce dernier facteur n'opère plus, et le sable est systématiquement déplacé de manière unidirectionnelle. De surcroît, le débouché de la buse se situant dans le secteur où aboutit la dérive littorale, tout le sable transporté de l'est vers l'ouest est repris à cet endroit et exporté vers la zone subtidale, ce qui explique que la plage ait bel et bien perdu l'essentiel de son stock de sable initial (fig. 4). Pour tenter de pallier les effets de la dérive littorale et dans l'espoir de piéger du sable à l'est de la plage, à la demande de riverains, deux épis transversaux ont été édifiés à la fin des années quatre-vingt dix et renforcés en 2004 (photo 1C). Bien évidemment, cette réalisation n'a eu aucun effet car, à cette époque, l'essentiel du stock de sable avait déjà disparu de ce secteur.

\section{Dynamique sédimentaire actuelle}

5 campagnes de levés topographiques ont été réalisées au DGPS de type Trimble 5700/5800. Les mesures ont été effectuées en mode RTK (Real Time Kinematics) avec une précision horizontale de $2 \mathrm{~cm}$ et verticale de 1,5 cm. Ces données ont permis de caractériser précisément la

Figure 4 : Dynamique hydrosédimentaire de la plage de Sainte-Anne

sedimentar dynamic of the Sainte Anne Beach

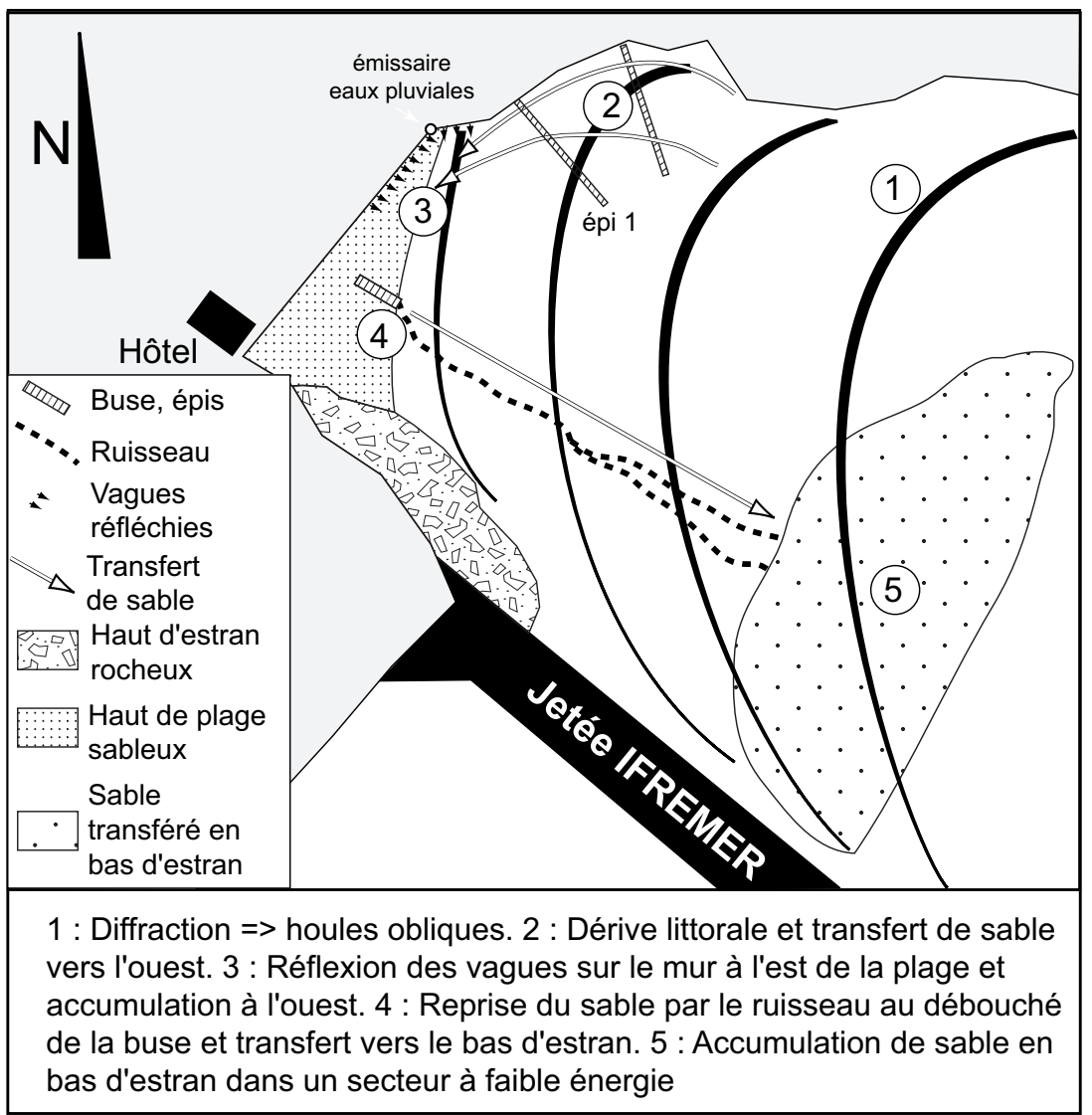


topographie et l'altimétrie de la plage d'une part et de quantifier les transferts sédimentaires sur une année entière, d'avril 2007 à mars 2008, d'autre part.

La figure 5, montre clairement que la plage, nettement décalée vers le sud ouest de l'anse, fait face à l'est et aux houles diffractées. Le seul endroit où elle dépasse le niveau des pleines mers de grandes vives eaux est au pied de l'hôtel au niveau de l'accès méridional (profil 4, fig. 6). Son altitude décroît ensuite régulièrement vers le nord est puisqu'elle n'atteint plus que 2,5 mètres au pied de l'accès nord et environ 2 mètres entre les deux épis (profil 0 et 1, fig. 6). Parallèlement, le profil du haut de plage, convexe au niveau des profils 2, 3 et 4 tend ensuite à s'aplatir puis à devenir concave. Du profil 4 au profil 0 la pente de la plage $(\tan \beta)$ au-dessus du niveau de la mimarée diminue régulièrement de 0,082 à 0,025 . Le débouché de la buse se situe exactement à la base de l'accumulation dans le secteur qui marque le terme de la dérive littorale (profil 3, fig. 6 et photo 2). On comprend donc que tout apport de sable à ce niveau est destiné à être exporté vers le bas de plage et, par là même, que depuis la construction de la jetée d'IFREMER, le sable du nord de la plage progressivement transporté vers le secteur d'accumulation est ensuite descendu vers le bas de l'estran d'où il ne peut remonter (fig. 4).

Les relevés effectués en 2007-2008, ne font que confirmer l'évolution mise ne évidence précédemment. Les déplacements de sédiments n'affectent que la partie sableuse de l'estran et sont en général de faible ampleur. Ainsi durant toute la période estivale (fig. 7A) l'absence de houles explique qu'il n'y ait ni dérive littorale ni réflexion sur les murs de haut d'estran. Par contre, les vents locaux de sud génèrent un clapot de périodes inférieures à 6 secondes (Guilcher et al., 1957) qui déferle parallèlement au trait de côte et entraîne une remontée de sable dans la partie centrale

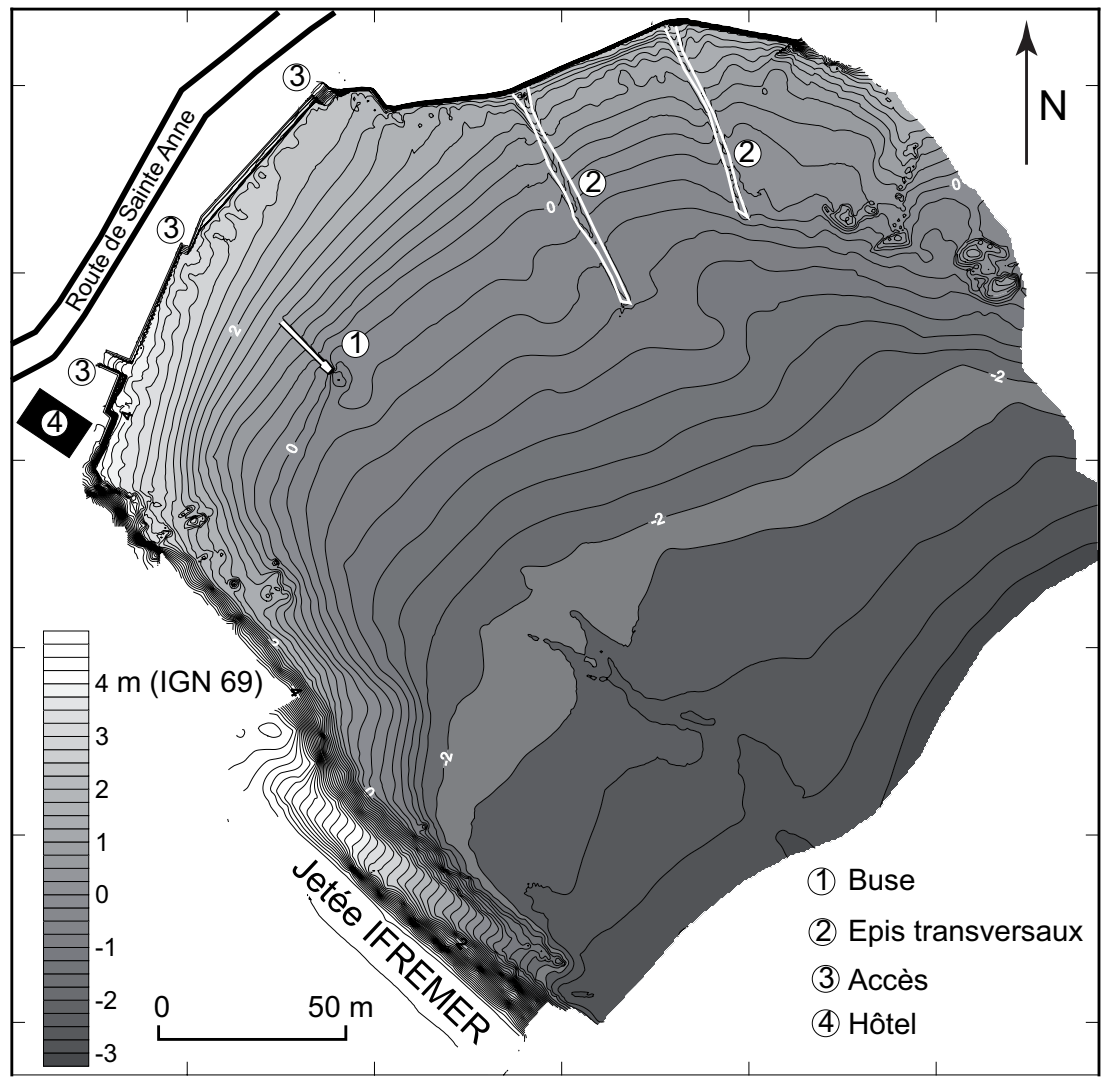

Figure 5 : Topographie de la plage de Sainte-Anne en avril 2007 phy of the SainteAnne Beach in april 2007 


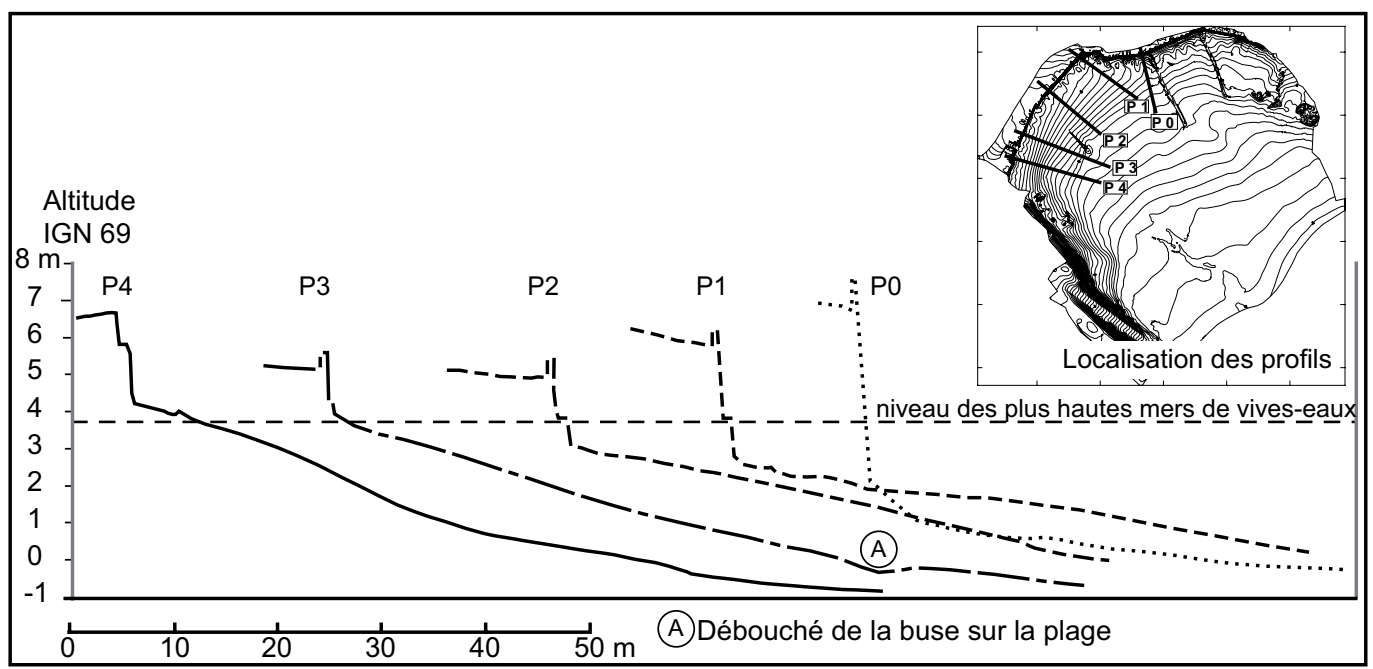

Figure 6: Profils de la plage de Sainte-Anne en avril 2007 Sainte-Anne beach profiles in April 2007

Photo 2 : Avril 2007, embouchure du ruisseau busé sur la plage. Le sable au terme de la dérive est exporté vers le bas d'estran April 2007, mouth of the canalized stream on the beach. The drift sand is transported toward the lower part of the beach

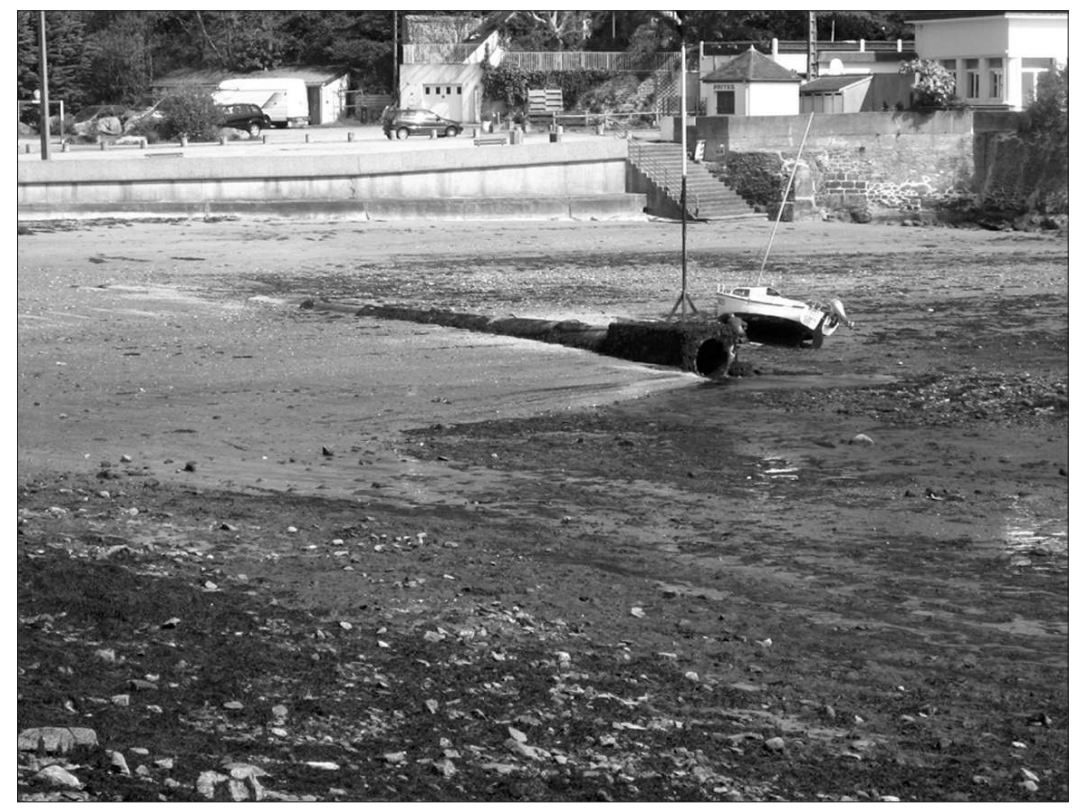

de la plage. Ce dépôt ne se fait pas aux dépens du bas d'estran mais à partir des secteurs attenants au-dessus du niveau de la mi-marée. Il n'y a donc pas d'engraissement à proprement parler. En l'absence de tempêtes automnales, cette dynamique se prolonge jusqu'en décembre (fig. 7B). Les conséquences des tempêtes hivernales sont bien plus importantes. Au cours du mois de janvier (fig. 7C) on note un départ de sable au pied du mur entre les deux accès septentrionaux et une tendance à l'accumulation vers le sud. Le dernier relevé, immédiatement postérieur à la très forte tempête du 10 mars qui a touché les côtes de Bretagne, montre nettement les effets cumulés de la réflexion des vagues sur les ouvrages et de la dérive littorale (fig. 7D). À l'est, la présence des 
épis n'entraîne aucune accumulation sédimentaire, ce qui est logique étant donné l'absence de sable en amont dérive. On remarque tout au plus un déplacement transversal de quelques mètres cubes de sédiment. À l'ouest, tout le haut de plage subit un démaigrissement important qui atteint 80 centimètres au pied de l'accès central. Tout le stock déplacé s'accumule au sud ouest et tend même à recouvrir en partie le secteur caillouteux situé au pied de la jetée. Au terme d'une année de relevés, le bilan confirme nettement la tendance évolutive générale depuis la construction de la jetée d'IFREMER, évolution qui voit la plage pivoter vers le sud ouest face aux houles diffractées (fig. 7E). Il semble évident qu'immédiatement après la construction de l'ouvrage, lorsque le stock de sable était plus important sur la plage les transferts ont du être beaucoup plus volumineux.
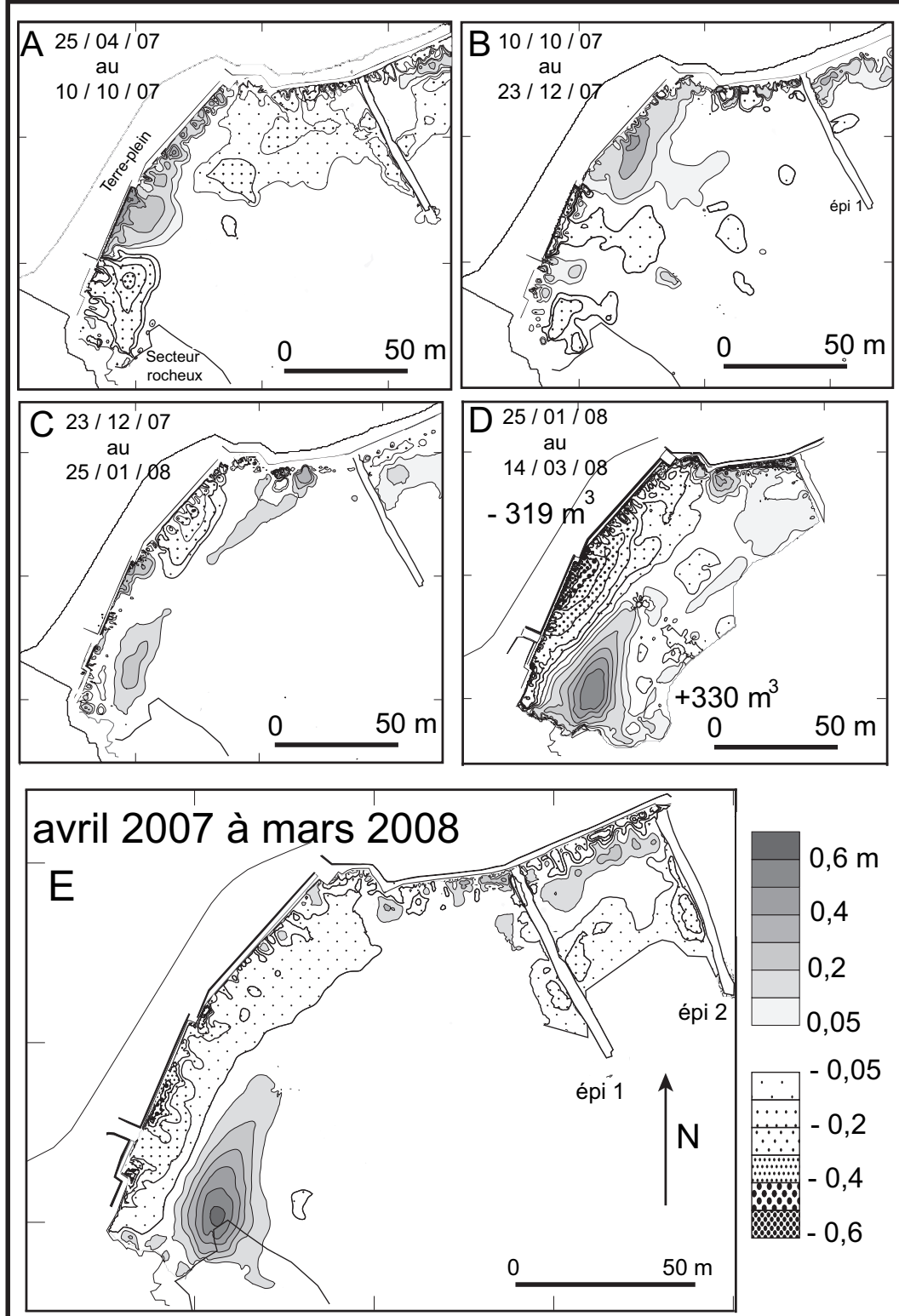

Figure 7 : Bilan des transferts sédimentaires d'avril 2007 à mars 2008 ment of sediment transfert from april 2007 to march 2008 


\section{Une opération de rechargement indispensable}

\section{MODIFICATIONS PRÉALABLES DES OUVRAGES EXISTANTS}

Le rechargement de la plage de Sainte-Anne va être mené dans un cadre contraignant qui n’a plus grand-chose de naturel et dont certains éléments ne peuvent être modifiés. Ainsi, la jetée d'IFREMER, à l'origine de la dérive littorale, a créé un abri qui a permis l'installation de dizaines de mouillages pour bateaux de plaisance. Les murs de haut de plage, qui réfléchissent la houle après déferlement, sont indispensables à la protection des terrasses, de la route, ainsi qu'un terreplein promenade. La destruction de ces ouvrages n'étant pas à l'ordre du jour l'opération de rechargement devra se faire dans ce contexte hydrodynamique. Jusqu'en 2007, toute opération de réensablement était vouée à un échec à court terme puisque le sable, où qu’il puisse être déposé, était destiné à migrer vers le débouché de la buse et à descendre ensuite vers la zone subtidale. En préalable à tout rechargement des modifications d'ouvrages ont été préconisées et pour certaines déjà réalisées. En octobre 2007 la buse a été rallongée de trente-cinq mètres et actuellement, son débouché se situe largement au-delà de la rupture de pente qui marque la base du haut de plage (photo 3). De cette manière l'exutoire du ruisseau ne constitue plus un point de départ pour les sédiments au terme de la dérive.

Photo 3 : Travaux d'allongement de la buse au débouché du ruisseau en septembre 2007

Lengthening of the pipe at the mouth of the stream in september 2007

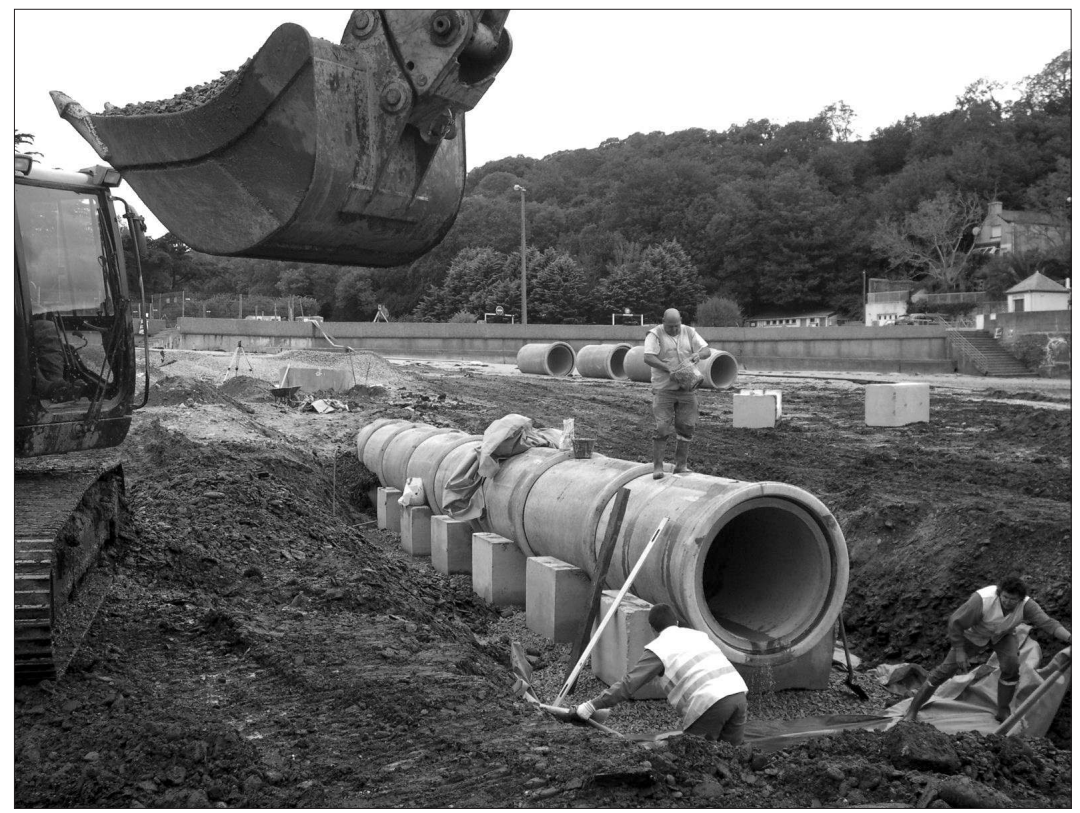

Dans le même esprit, le débouché des eaux pluviales situé au pied de l'accès oriental de la plage occasionnait un transport de sable vers le bas d'estran lors des fortes précipitations. Il a été raccordé au réseau de collecte existant sur le secteur.

Dans un futur proche, l'étanchéité de l'épi $n^{\circ} 1$ sera renforcée. Ce dernier dont la fonction est de bloquer du sable sur l'est de la plage est actuellement perméable, car constitué d'un amas de gros blocs présentant de larges vides interstitiels. Ces vides devront être colmatés par des blocs et cailloux plus petits collectés sur place.

Pour autant, en elles-mêmes ces opérations ne peuvent, contrairement à l'attente de certains élus, s'accompagner de remontée naturelle du sable et le rechargement s'avère indispensable. 


\section{DÉTERMINATION DES VOLUMES NÉCESSAIRES ET DES STOCKS DISPONIBLES}

L'étape suivante du travail a consisté à déterminer les volumes de sable nécessaires au rechargement du haut de plage, et à vérifier si ces stocks étaient disponibles sur place et d'une granulométrie compatible avec celle du haut de l'estran.

Deux secteurs de rechargement ont été définis. Le premier se situe entre les accès à la plage et le second en amont dérive de l'épi $n^{\circ} 1$. Dans le premier cas, pour que le sable puisse se maintenir en place, il faut que partout le niveau du haut de plage et le profil de l'estran soient les mêmes qu'au pied de l'hôtel (fig. 6, profil 4). L’objectif, ici, est que tout du long du mur de défens, le niveau de la plage dépasse celui des plus hautes mers (environ $4 \mathrm{~m}$ ), afin d'empêcher la réflexion des vagues contre l'ouvrage. À l'est, au niveau des épis, il est impossible de recharger suffisamment pour atteindre cette altitude. En effet, la côte maximale de l'épi 1 est à 2,90 m soit plus d'un mètre sous le niveau des plus hautes mers. À cet endroit nous avons donc envisagé un rechargement ne dépassant pas cette côte, avec une pente du même type qu'à l'ouest. Dans ce secteur, et sans doute très rapidement après l'opération, la réflexion des vagues sur la base des murs étalera le sable vers le milieu de l'estran mais il restera, en partie au moins, bloqué à l'est des épis. Ceci reviendra à reconstituer ici un estran sableux submersible là où à l'heure actuelle ne subsistent que des cailloux (photo 4A). Il faut noter que cette partie de la plage, avant même la construction de la jetée, était recouverte à toutes les hautes mers (photo 4B).
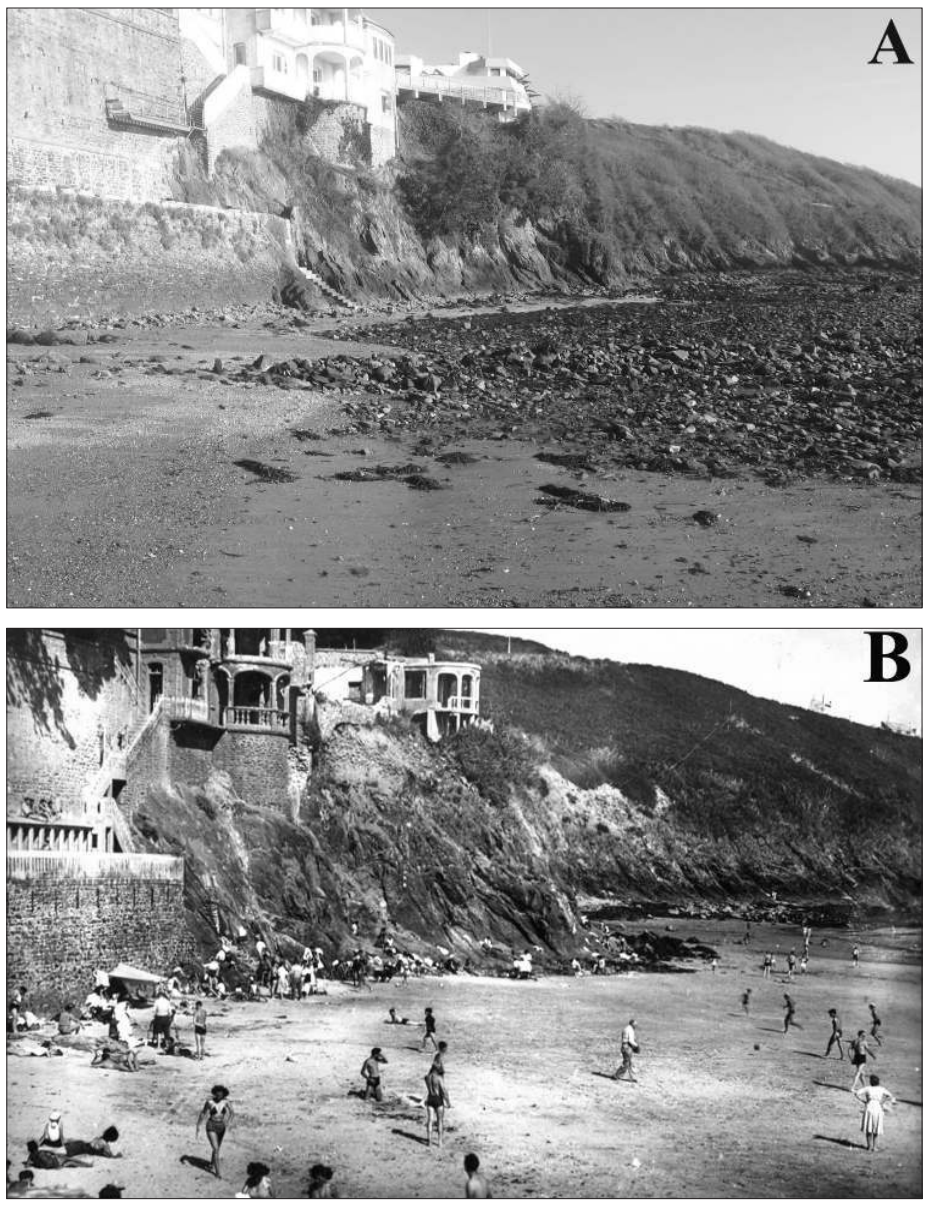

Photo 4 : Partie orientale de la plage de Sainte-Anne : (A) en 2008, (B) vers 1950

Eastern part of the beach of sainte-Anne: (A) in 2008, (B) circa 1950 
La simulation de la topographie post-rechargement a été réalisée par numérisation à l'aide du logiciel Surfer 8. La superposition de la topographie actuelle et de celle qui a été modélisée pour l'avenir a permis de déterminer le volume de rechargement recherché qui est de $1100 \mathrm{~m}^{3}$ (fig. 8).

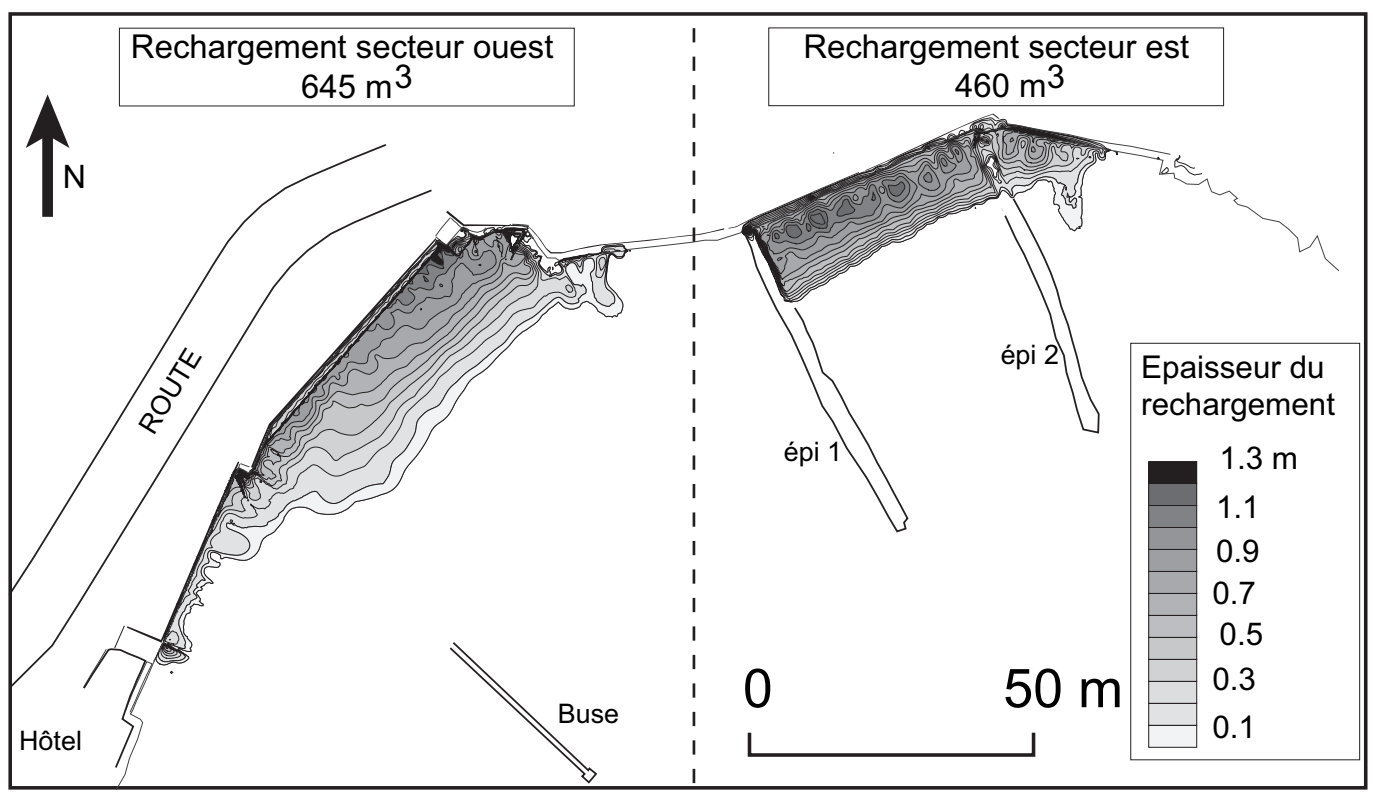

Figure 8 : Simulation des volumes de rechargement de la plage Simulation of the volumes of beach replenishment

Ensuite, des secteurs de prélèvement potentiel ont été recherchés. Sous la côte -2,25 m, c'est-àdire sous le niveau des basses mers de mortes eaux moyennes et jusqu'au-delà du niveau des plus basses mers possibles, tout le bas d'estran est constitué d'un banc de sable (fig. 9). Le nord est du banc étant colonisé par un herbier à zoostères, il ne peut être envisagé de l'utiliser comme secteur de prélèvement. Lors d'une basse mer de la marée de vive-eau de coefficient 108 du 26 octobre 2007, des sondages ont été effectués à la tarière sur le reste du secteur. Ils ont permis de mesurer l'épaisseur du dépôt qui dépasse partout $50 \mathrm{~cm}$ sur les $4400 \mathrm{~m}^{2}$ découverts à cette date. Ceci représente un volume potentiel minimal de $2200 \mathrm{~m}^{3}$. Dans chaque sondage ainsi que sur le haut d'estran, des échantillons ont été récoltés et analysés afin de vérifier si le sable du banc de bas de plage pouvait être utilisé pour le rechargement. Dans les secteurs d'accumulation du haut d'estran, au pied du mur ou en amont des épis, le sédiment est très homométrique constitué de 65 à $95 \%$ de sable fin. Sable moyen et sable fin constituent plus de $95 \%$ du stock sédimentaire qui est totalement dépourvu de pélites, de sable grossier ou de graviers (fig. 9, échantillons 2, 5, 6). En bas de plage le sédiment est de même type puisque sable fin et moyen cumulés représentent partout plus de $70 \%$ du stock (fig. 9, échantillons 21 à 33). Le tri est toutefois moins bon et tous les échantillons contiennent à la fois des pélites (6\% en moyenne) et des sables grossiers voire des graviers. Cette fraction grossière est à la fois biogène, produite sur place par les bivalves, et terrigène. Dans ce cas elle provient sans doute en partie des apports du bassin-versant du ruisseau. Le grain moyen des échantillons est aussi comparable, compris entre 130 et $250 \mu \mathrm{m}$ en haut, et entre 170 et $319 \mu \mathrm{m}$ en bas d'estran. 


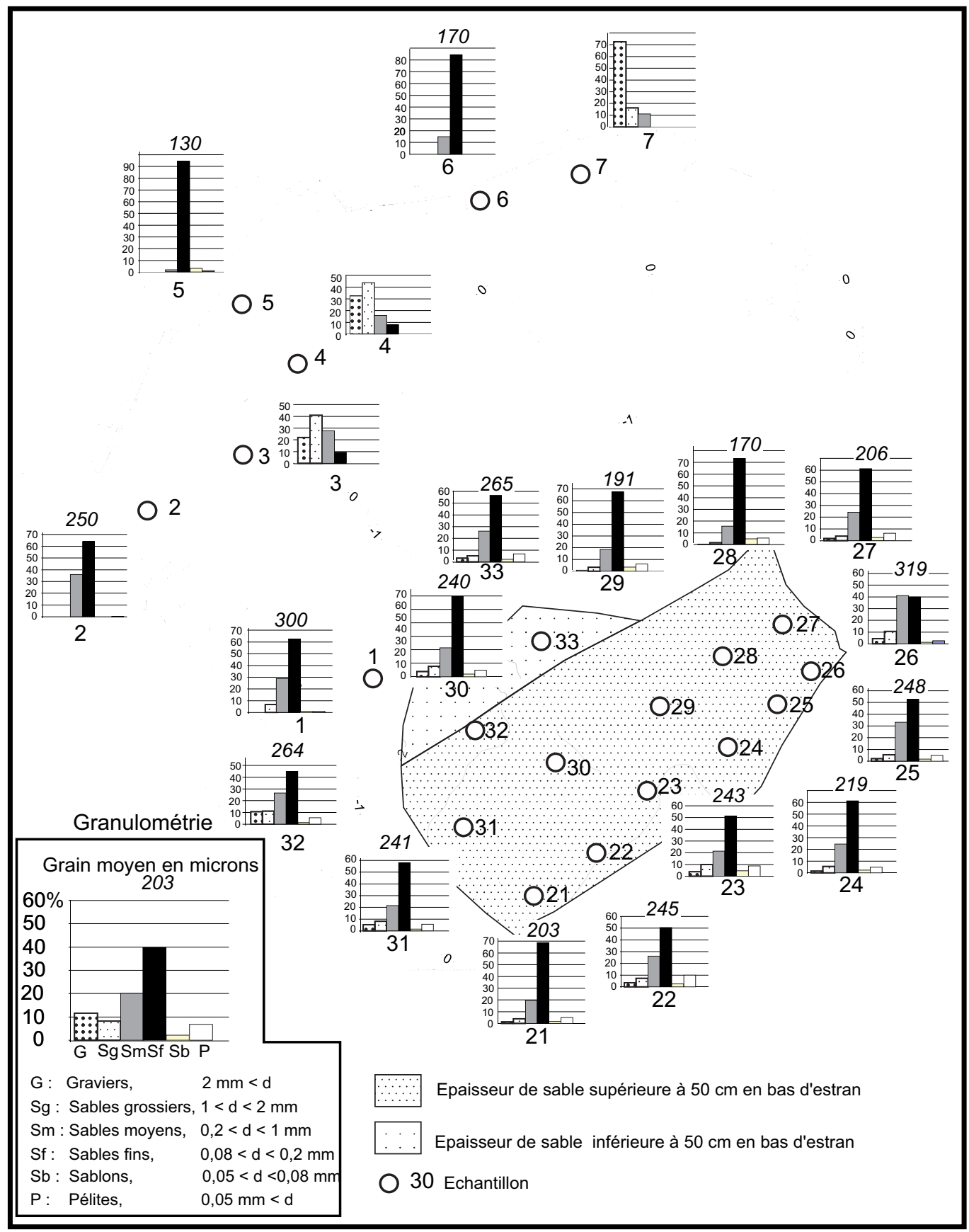

Figure 9 : Granulométrie des sédiments de la plage et localisation du secteur de prélèvement. Granulometry of sediment and location of extraction area

Ces résultats n’ont rien de surprenant et viennent conforter ce qui a été démontré précédemment, à savoir que ce stock de sable provient pour l'essentiel de l'érosion du haut de plage depuis qu'a été construite la jetée d'IFREMER. Le rechargement consistera donc simplement à remonter 
une partie du stock sédimentaire vers le haut de plage. Il sera nécessaire de remonter plus que les $1100 \mathrm{~m}^{3}$ requis, sachant que $6 \%$ du stock transféré est constitué de pélites qui ne pourront se maintenir dans les conditions d'agitation qui caractérisent le haut de plage. Dans le cahier des charges des travaux à entreprendre nous avons spécifié qu'un transfert minimal de $1200 \mathrm{~m}^{3}$ était nécessaire, ce qui n'exclut pas bien sûr d'envisager des quantités plus importantes, le stock disponible étant au minimum de $2200 \mathrm{~m}^{3}$. Toutefois cet aspect du problème ne se règlera que lors des travaux. En effet, pour des raisons financières et au vu des quantités limitées qui sont en jeu, le prélèvement et le transport du sable se fera à basse mer, avec l'aide de pelles mécaniques, chargeurs, tracteurs ou camions et non pas par dragage et refoulement. L'opération ne pouvant être réalisée que par très grand coefficient supérieur à 105, le chantier ne sera opérationnel que pendant 3 à 4 heures au moment de la basse mer et sur deux ou trois jours au maximum. Dans ces conditions, c'est l'organisation du chantier qui constituera le facteur limitant.

\section{Conclusion}

Notre approche du problème posé par le déséquilibre sédimentaire de la plage de Sainte-Anne se veut délibérément environnementaliste et gestionnaire. À une solution facile qui aurait consisté en un rechargement massif par du sable prélevé sur des gisements sous-marins naturels nous avons préféré une solution basée sur la compréhension fine de la dynamique hydrosédimentaire locale dans un cadre contraint par une série d'aménagements inadéquats. Tous les ouvrages progressivement mis en place au cours du XXe siècle ont contribué au transfert du sable du haut de plage vers le bas de la zone intertidale. C'est ce sable qui va être utilisé pour le rechargement. La plupart des ouvrages (jetée, murs de haut de plage) sont destinés à rester en place il est évident que leur impact sur l'hydrodynamisme local restera inchangé. Par exemple, la dérive littorale générée par la présence de la jetée continuera de transporter le sable du nord est vers le sud ouest de la plage. Trois mesures essentielles toutefois devraient permettre de ralentir fortement le désensablement du haut de l'estran. Le rechargement jusqu'à une altitude supérieure à celle des plus hautes mers limitera la réflexion des vagues sur le mur du haut de plage. Le rallongement de la buse canalisant le débouché du ruisseau empêchera la réexportation du sable vers le bas de la plage et l'existence d'épis favorisera la stabilisation du sable rapporté à l'est. Toutefois la situation ne sera pas stable. En effet le sable tendra de toutes façons à descendre vers le bas de l'estran. Lors du jusant, l'égouttage du haut de plage et l'écoulement de la nappe phréatique emportent le sable fin. À l'occasion de fortes tempêtes associées à de forts coefficients, même dans le cadre très abrité de l'anse de Sainte-Anne les vagues peuvent déferler largement au-dessus du niveau des plus hautes mers et entraîner un aplatissement du profil de l'estran.

Il est donc nécessaire de prévoir un suivi de la situation dont le pas de temps reste à définir. Ce suivi basé sur des levés topographiques permettra d'estimer précisément les changements intervenus et de calibrer les opérations de réajustement.

\section{Bibliographie}

Berg (D.-W.), Duane (D.-B.), 1968. - Effect of particule size and distribution on stability of artificially filled beach. Presque Isle Peninsular, Pennsylvania, Proceedings of the $11^{\text {th }}$ Conference on Great Lakes research, Michigan, p. 161-178.

Capobianco (M.), Hanson (H.), Larson (M.), Steetzel (H.), Stive (M. J. F.), Chatelus (Y.), Aarninkhof (S.), Karambas (T.), 2002. - "Nourishment design and evaluation: applicability of model concepts", Coastal Engineering, vol. 47, n² 2, p. 113-135.

Cohen (O.), Anthony (E. J.), 2007. - "Gravel beach erosion and nourishment in Nice, French Riviera", Méditerranée, $\mathrm{n}^{\circ} 108$, p. 99-103. 
DiXon (K. W.), Pilkey (O.-H.), 1989. - Beach replenishment on the U.S. Gulff Mexico shoreline, Proceedings of Coastal Zone'89 Conference, New York, p. 2007-2020.

Fichaut (B.), Suanez (S.), Prigent (M.), 2008. - « Suivi morpho-sédimentaire de la plage de Sainte Anne du Portzic (Communes de Brest et Plouzané) dans le cadre des opérations de réhabilitation », rapport d'expertise scientifique, GEOMER-LETG UMR 6554 CNRS, IUEM/BMO, 23 p.

FINKL (C. W.), 1996. - "What might happen to America's shoreline if artificial beach replenishment is curtailed: a prognosis for Southeastern Florida and outer sandy regions along regressive coasts", Journal of Coastal Research, vol. 12, n 1 , p. III-IX.

FinkL (C. W.), Walker (H. J.) 2005. - "Beach nourishment”, dans Schwartz (M. L.) (dir.), Encyclopedia of Coastal Science, Springer, The Netherlands, p. 147-161.

French (P. W.), 2001. - «Coastal defences : processes, problems and solutions », Londres, Routledge, 266 p. Guilcher (A.), Vallantin (P.), Angrand (J.-P.), Gallois (P.), 1957. - « Les cordons littoraux de la rade de Brest », Cahiers Océanographiques et d'Étude Côtière, n 1, p. 21-54.

Hamm (L.), Capobianco (M.), Dette (H. H.), Lechuga (A.), Spanhoff (R.), Stive (M. J. F.), 2002. - "A summary of European experience with shore nourishment", Coastal Engineering, vol. 47, n² 2, p. 237 264.

Hanson (H.), Brampton (A.), Capobianco (M.), Dette (H. H.), Hamm (L.), Laustrup (C.), Lechuga (A.), SpANhoff (R.), 2002. - "Beach nourishment projects, practises and objectives - a european overview", Coastal Engineering, vol. 47, $\mathrm{n}^{\circ}$ 2, p. 81-111.

Hallégouet (B.), Guilcher (A.), 1990. - « Moulin Blanc artificial beach, Brest, western Brittany, France », Journal of Coastal Reseach, Special Issue n 6, p. 17-20.

Leonard (L.-A.), Clayton (T.), Pilkey (O. H.), 1990a. - « An analysis of replenished beach design parameters on US east coast barrier islands ", Journal of Coastal Reseach, vol. 6, n 1, p 15-36.

Leonard (L. A.), Dixon (K. L.), Pilkey (O. H.), 1990b. - «A comparison of beach replenishment on the us Atlantic, Pacific and Gulf Coasts », Journal of Coastal Research, Special Issue n 6, p. 127-140.

Matias (A.), Ferreira (O.), Mendes (I.), Dias (J. A.), Vila-Concejo (A.), 2005. - « Artificial construction of dunes in the South of Portugal », Journal of Coastal Research, vol. 21, n 3, p. 472-481.

Newman (D. E.), 1976. - Beach replenishment: sea defenses and a review of the artificial beach replenishment, Proceedings of the Institution of Civil Engineers, vol. 6, n 60, p. 45-60.

Pilkey (0. H.), Clayton (T. D.), 1987. - Beach replenishment: the national solution?, Proceedings of Coastal Zone' 87, New York, p. 1408-1419.

Pilkey (0. H.), 1990. - «A time to look back at beach replenishment », Journal of Coastal Research, vol. 6, $\mathrm{n}^{\circ} 1$, p. III-VII.

Pupier-Dauchez (S.), 2002. - Le rechargement sédimentaire: De la défense des côtes à l'aménagement du littoral (Analyse des pratiques sur la côte Atlantique française), thèse de doctorat en géographie, Université de Bretagne Occidentale, 2 tomes, $510 \mathrm{p}$.

Rogers (S.M. JR.), 2000. - Beach nourishment for hurricane protection: North Carolina project performance in Hurricanes Denis and Floyd, Proc., National Beach Preservation Conference, American Shore and beach Preservation Association, Maui, Hawaii, p. 7-10.

SAUM, 1997. - Schéma d'Aptitude et d'Utilisation de la Mer (SAUM) : Rade de Brest - 1977 Étude analytique et méthodologique, Direction Départementale de l'Équipement, Département du Finistère (Quimper), $169 \mathrm{p}$.

VAN DER WAL (D.), 2004. - Beach-dune interactions in nourishment areas along the Dutch coast, Journal of Coastal Research, vol. 20, $\mathrm{n}^{\circ}$ 1, 317-325.

Verhagen (H.-J.), 1996. - «Analysis of beach nourishment schemes », Journal of Coastal Reseach, vol. 12, $n^{\circ} 1,179-185$.

Cet article a été reçu le 3 juin 2008 et définitivement accepté le $1^{\text {er }}$ octobre 2008. 